\title{
HDAC2 attenuates TRAIL-induced apoptosis of pancreatic cancer cells
}

\author{
Susanne Schüler ${ }^{1}$, Petra Fritsche1, Sandra Diersch¹, Alexander Arlt², Roland M Schmid', Dieter Saur ${ }^{1}$ and \\ Günter Schneider*1
}

\begin{abstract}
Background: Pancreatic ductal adenocarcinoma (PDAC) is one of the most malignant tumors with a dismal prognosis and no effective conservative therapeutic strategies. Although it is demonstrated that histone deacetylases (HDACs), especially the class I HDACs HDAC1, 2 and 3 are highly expressed in this disease, little is known about HDAC isoenzyme specific functions.

Results: Depletion of HDAC2, but not HDAC1, in the pancreatic cancer cell lines MiaPaCa2 and Panc1 resulted in a marked sensitization towards the tumor necrosis factor-related apoptosis-inducing ligand (TRAIL). Correspondingly, the more class I selective HDAC inhibitor (HDACl) valproic acid (VPA) synergized with TRAIL to induce apoptosis of MiaPaCa2 and Panc1 cells. At the molecular level, an increased expression of the TRAIL receptor 1 (DR5), accelerated processing of caspase 8 , pronounced cleavage of the $\mathrm{BH} 3$-only protein Bid, and increased effector caspase activation was observed in HDAC2-depleted and TRAIL-treated MiaPaCa2 cells.
\end{abstract}

Conclusions: Our data characterize a novel HDAC2 function in PDAC cells and point to a strategy to overcome TRAIL resistance of PDAC cells, a prerequisite to succeed with a TRAIL targeted therapy in clinical settings.

\section{Background}

The incidence of pancreatic ductal adenocarcinoma (PDAC) is only about 10 in $10^{5}$, but it is the fourth leading cause of cancer-related death with a 5-year survival rate beyond 5\% [1]. As there is no significant improvement in patient survival over the last decades [2] and biologicals like the epidermal growth factor receptor (EGFR) inhibitor erlotinib are only active in a subset of patients [3], there is a need to develop new rational based therapeutic strategies in preclinical settings.

Histone deacetylases (HDACs) deacetylate the $\varepsilon$-amino group of lysines located at the $\mathrm{N}$-terminal tail of histones, which leads to a repressive chromatin formation (heterochromatin) and the suppression of gene expression [4]. In addition to the condensation of chromatin, HDACs deacetylate various proteins to regulate their function. Many of these proteins are transcription factors, such as $\mathrm{p} 53, \mathrm{C} / \mathrm{EBP} \beta, \mathrm{NF}-\mathrm{kB}$ and STATs. Therefore changes in the transcriptome upon HDAC inhibitor (HDACI) treat-

* Correspondence: guenter.schneider@lrz.tum.de

1 Technische Universität München, Klinikum rechts der Isar, II. Medizinische Klinik, Ismaninger Str. 22, 81675 Munich, Germany

Full list of author information is available at the end of the article ment can be due to a direct modulation of the "histone code" or the consequence of a rather indirect modulation of signaling pathways and transcription factor activities [5-7]. The eighteen deacetylases encoded in the mammalian genome are grouped into class I (HDAC 1, 2, 3 and 8), class II (HDAC 4, 5, 6, 7, 9 and 10), class III (SIRT 1-7) and class IV (HDAC11) enzymes [4]. In tumors, HDACs are involved in the regulation of proliferation, apoptosis, differentiation, migration and angiogenesis [8] and are hence promising targets for therapeutic intervention. In PDAC, the contribution of HDACs towards the control of proliferation, apoptosis and metastasis is clearly documented [9]. Consistently, various HDACI were developed over the last years and are now tested in numerous clinical trials [10]. However, HDACI as monotherapeutics are only effective in a defined subset of hematological tumors and there are several evidences that rational- and molecular-defined HDACI-based combination therapies are more useful for the treatment of solid cancers [11]. Defining suitable HDACI-based combinations is especially important in PDAC since a recent phase II clinical trial failed to demonstrate effectiveness of the weak HDACI 
CI-994 combined with the current standard chemotherapeutic gemcitabine [12].

In this study we show that specific depletion of HDAC2, but not HDAC1, sensitizes PDAC cells towards tumor necrosis factor-related apoptosis-inducing ligand (TRAIL)-induced apoptosis, suggesting a new therapeutic strategy.

\section{Results \\ HDAC2 depletion sensitizes PDAC cells towards TRAIL- induced apoptosis}

We recently observed the HDAC2 mediated control of the DNA-damage response in PDAC cells [13]. To investigate HDAC2 function in the extrinsic apoptotic pathway, we used HDAC2-specific siRNA in PDAC cells (figure 1A). As shown in figure 1B, HDAC2-depleted $\mathrm{MiaPaCa} 2$ and Panc1 cells revealed a distinctly decreased viability after the treatment with TRAIL as compared to control siRNA transfected cells. Consistently, the TRAILinduced apoptotic fraction was significantly increased in a dose-dependent manner in HDAC2-depleted $\mathrm{MiaPaCa} 2$ and Panc1 cells (figure $1 \mathrm{C}$ ). Increased apoptosis induction by TRAIL in MiaPaCa2 and Panc1 cells was further validated using western blots for cleaved PARP (figure 1D). In addition, increased PARP cleavage in HDAC2 siRNA transfected DanG and BxPc3 cells was observed, arguing for a general control of extrinsic apoptotic signaling by HDAC2 in PDAC cells (data not shown).

\section{Valproic acid sensitizes PDAC cells towards TRAIL-induced apoptosis}

Since valproic acid (VPA) is a more class I specific HDACI and known to deplete HDAC2 via a proteasomal pathway $[14,15]$, we validated the results obtained with functional genomics using VPA. We used VPA at a concentration of $1.5 \mathrm{mM}$, which is achievable in therapeutical settings and has no influence on PDAC cell proliferation or viability [13]. Co-treatment of MiaPaCa2 and Panc1 cells with TRAIL and VPA lead to a significantly reduced viability in a dose-dependent fashion, compared to cells treated with TRAIL alone (figure 2A). In line, an increased apoptotic fraction was observed in VPA and TRAIL co-treated MiaPaCa2 and Panc1 cells (figure 2B), reproducing the results obtained with RNA interference (RNAi) and suggesting that HDAC2 attenuates TRAIL-induced apoptosis in PDAC cells.

\section{TRAIL mediated apoptosis is not influenced by HDAC1 in PDAC cells}

Since HDAC-dependent non-redundant functions are ill defined, we tested whether HDAC1-depletion also sensitizes for TRAIL-induced apoptotsis. Transfection of HDAC1 siRNA into MiaPaCa2 and Panc1 cells resulted in a specific knockdown of HDAC1, whereas HDAC2 expression was not affected (figure 3A). Nevertheless, TRAIL-induced loss of viability of MiaPaCa2 and Panc1 cells was not changed in HDAC1 siRNA transfected cells compared to control siRNA transfected cells (figure 3B). Furthermore, no increase in the apoptotic fraction was observed in HDAC1-depleted and TRAIL-treated MiaPaCa2 and Panc1 cells in comparison to control siRNA transfected cells (data not shown). This argues that HDAC2 is specifically involved in the regulation of TRAIL induced apoptosis, independently of HDAC1.

\section{HDAC2-dependent regulation of the BH3-only protein NOXA is not involved in HDAC2-depletion mediated TRAIL sensitization}

Recently we observed that depletion of HDAC2 results in an increased expression of the pro-apoptotic BH3-only protein NOXA in PDAC cells [13]. To investigate the influence of NOXA on the HDAC2-dependent sensitization towards TRAIL-induced apoptosis, we simultaneously transfected HDAC2- and NOXA-specific siRNAs into $\mathrm{MiaPaCa} 2$ cells. As shown in figure 4A, upregulation of NOXA mRNA by the depletion of HDAC2 was significantly inhibited, when the cells were transfected with both siRNAs. Furthermore, HDAC2-depletion mediated sensitization of MiaPaCa2 cells towards TRAIL-induced apoptosis was not changed in HDAC2 and NOXA siRNA co-transfected cells (figure 4B), demonstrating that HDAC2-mediated sensitization towards death receptor induced apoptosis is independent of NOXA.

\section{HDAC2 regulates the TRAIL receptor DR5 in MiaPaCa2 cells}

Recent evidence suggests that HDACs can alter gene expression by the direct deacetylation of $\varepsilon$-amino-lysin groups at the $\mathrm{N}$-terminal tails of histones and/or by the direct influence on various signaling pathways [6]. To discriminate between both possibilities we transfected MiaPaCa2 cells with a control and a HDAC2-specific siRNA and treated the cells with TRAIL or a combination of TRAIL and actinomycin D to inhibit TRAIL signalinginduced transcription. As shown in figure 5A, TRAILinduced caspase $3 / 7$ activation was significantly increased in HDAC2-depleted MiaPaCa2 cells. Furthermore, blocking transcription in control and HDAC2 siRNA transfected MiaPaCa2 cells resulted in an increased caspase 3/7 activity, arguing that the TRAILinduced transcription functions mainly to restrain deathreceptor-induced apoptosis. Nonetheless, caspase 3/7 activity was significantly higher in TRAIL and actinomycin D co-treated HDAC2-depleted MiaPaCa2 cells than in TRAIL and actinomycin D co-treated control siRNA transfected cells, indicating that a change in the gene expression after HDAC2 depletion or a mechanism not requiring transcription, independently of TRAIL- 




induced transcription, at least contributes to the increased sensitivity of HDAC2-depleted MiaPaCa2 cells to TRAIL. In line with the increased activity of caspase 3/ 7 we observed an accelerated cleavage of caspase 8 in HDAC2-depleted and TRAIL-treated MiaPaCa2 cells, which correlated with the appearance of cleaved PARP as an indirect measurement of effector caspase activity (figure $5 \mathrm{~B}$ and $5 \mathrm{C}$ ). Similar results were observed in VPA treated MiaPaCa2 cells (data not shown). Furthermore, we detected a distinctly increased cleavage of Bid in HDAC2-depleted and TRAIL-treated MiaPaCa2 cells, compared to control siRNA transfected cells (figure 5D). In transcriptome profiles of HDAC2-depleted PDAC cells, which we have recently published [13], the TRAIL receptor DR5 was 2.2 fold upregulated in MiaPaCa2 cells and marginally increased in Panc1 cells. As shown in figure 5D and 5E, increased DR5 protein expression was detected in HDAC2-depleted MiaPaCa2 cells. In contrast, no change in the expression of DR4, c-Flip, XIAP, cIAP1, cIAP2, mcl1 or survivin was observed in HDAC2depleted MiaPaCa2 cells (figure 5D). A slight but consistent upregulation of $\mathrm{bcl}_{\mathrm{XL}}$ was observed in HDAC2- 


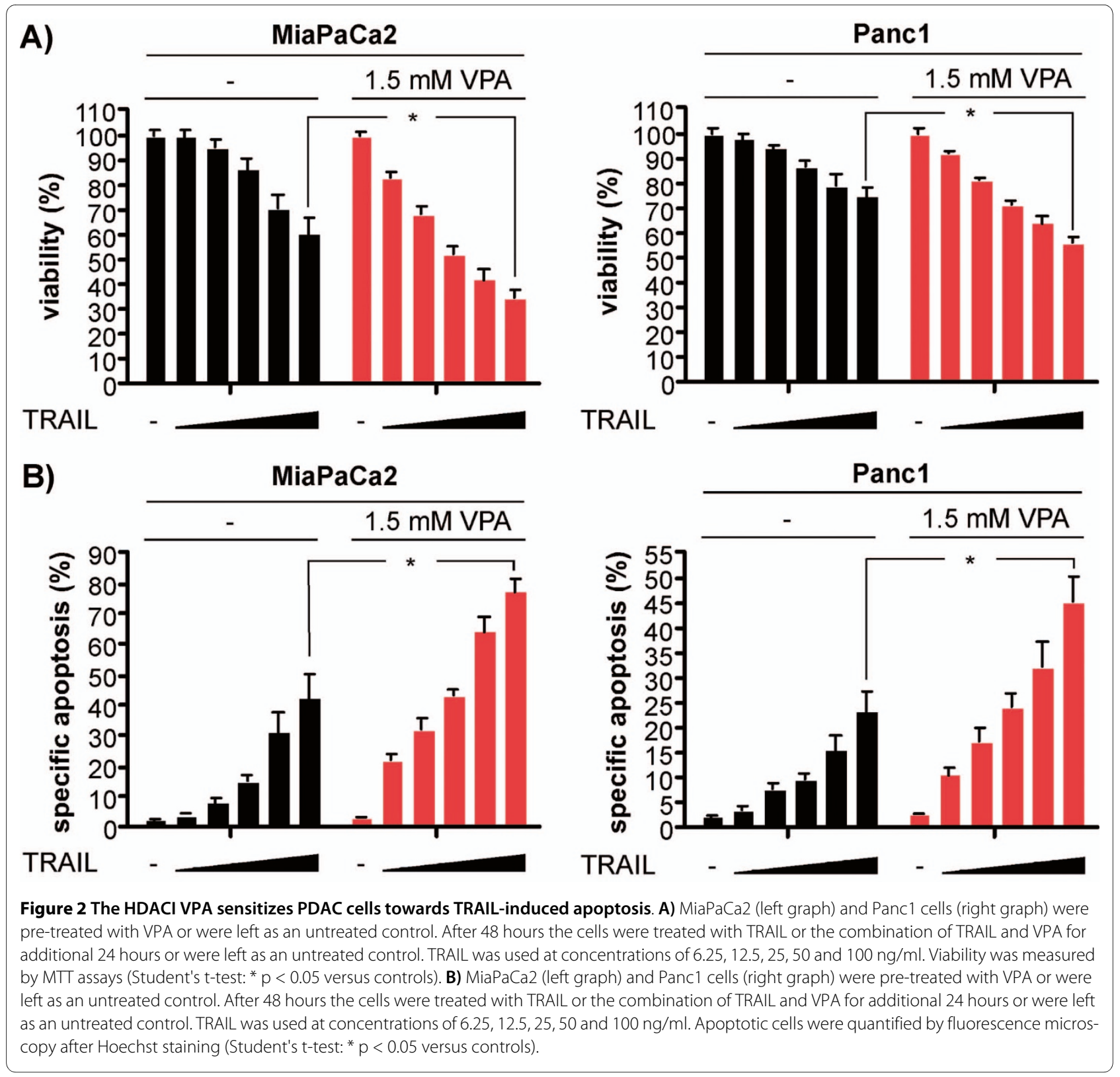

depleted MiaPaCa2 cells (figure 5D). Fitting to an increase in apoptosis induced by TRAIL in HDAC2depleted MiaPaCa2 cells, we observed a more pronounced elimination of c-Flip after the TRAIL treatment in HDAC2 siRNA transfected MiaPaCa2 cells (figure 5D). In contrast to $\mathrm{MiaPaCa} 2$ cells, upregulation of $\mathrm{DR} 5$ protein expression after HDAC2 depletion was not observed in Panc1 cells (figure 5E). Consistently, DR5 cell surface expression was slightly increased in $\mathrm{MiaPaCa} 2$ cells, but not in Panc1 cells (figure 5F). Together, these data argue that HDAC2-dependent sensitization towards TRAILinduced apoptosis works upstream of Bid in $\mathrm{MiaPaCa} 2$ cells.

\section{Discussion}

Deregulation of apoptosis is a hallmark of cancer and is an important cause of therapeutic failure of conventional chemotherapeutics $[16,17]$. Apoptosis can be initiated by the extrinsic death receptor-dependent as well as the intrinsic mitochondrial pathway and various mechanisms of resistance working on several levels were described in PDAC [18,19]. Triggering of death-receptors, like the CD95 receptor or TRAIL receptors, results in the activation of the initiator caspase 8 via the death-inducing signaling complex (DISC) with subsequent activation of effector caspases. In PDAC cells, which are type II cells, active caspase 8 cleaves the $\mathrm{BH} 3$-only protein Bid, which 


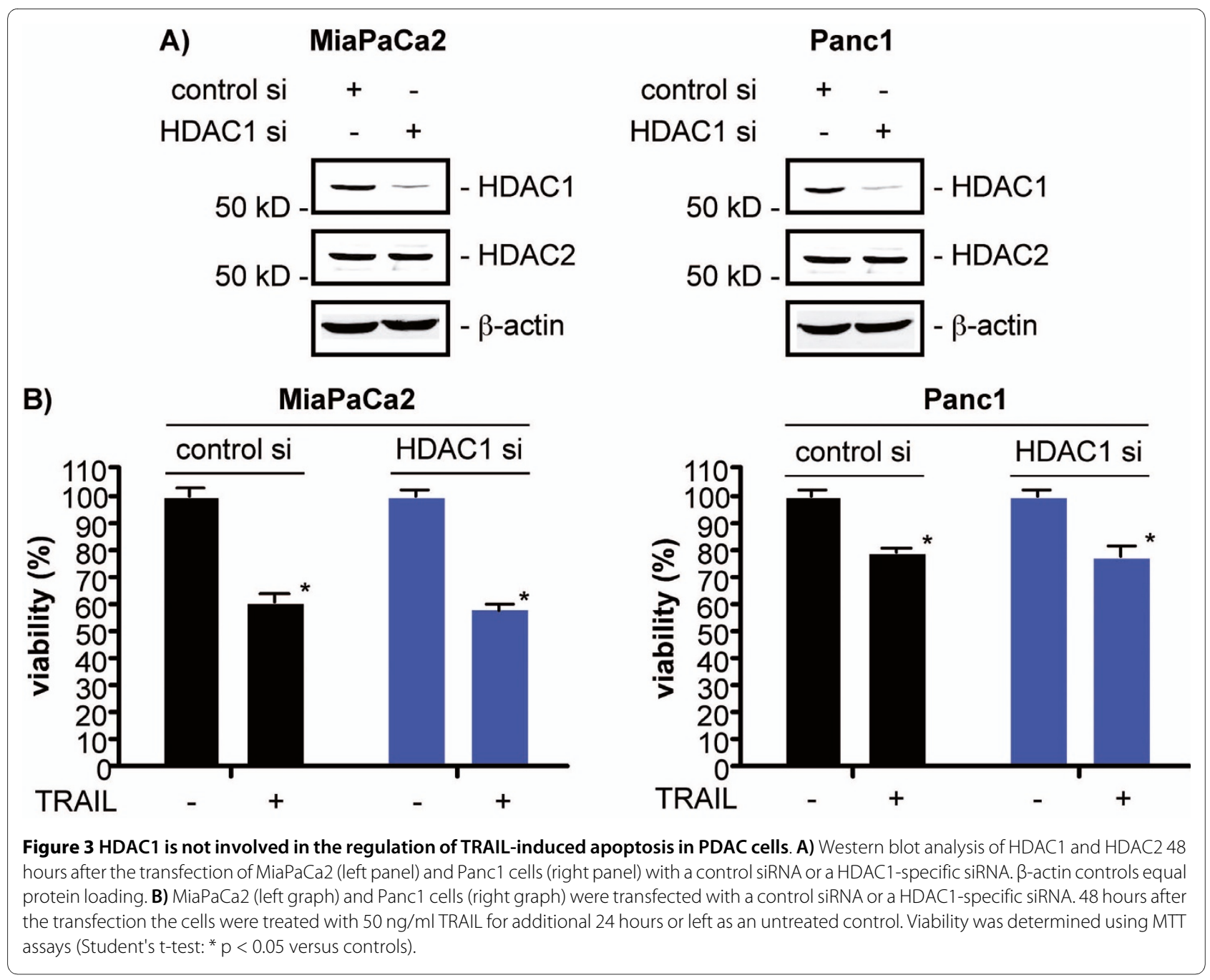

augments the activation of the caspase cascade via the mitochondrium [20,21]. Due to their selective toxicity towards cancer cells, TRAIL receptor agonists are promising cancer therapeutics and currently tested in clinical trials [21]. Furthermore, the observation that high TRAIL expression was correlated with an increased apoptotic index in the human pancreas argues that a TRAIL-based therapy might be a feasible strategy for the treatment of PDAC [22,23]. However, human primary tumor cells often resist TRAIL-induced apoptosis and PDAC cells reveal a relatively high half maximal inhibitor concentration (IC50) for TRAIL although they express the TRAIL receptors DR4 (TRAIL-R1) and DR5 (TRAIL-R2) as well as relevant mediators of the TRAIL receptor signaling pathway [24-26]. Therefore, strategies to sensitize PDAC cells towards TRAIL and to counteract resistance mechanisms are of value for defining new therapies for the treatment of PDAC.

In this study we show that the more class I selective HDACI VPA sensitizes PDAC cells towards TRAIL- induced apoptosis. Furthermore, we demonstrate that HDAC2, which is highly expressed in human PDACs $[13,27]$, controls resistance towards TRAIL. These results in combination with the high expression of HDAC2 in many solid tumors [28] and the recent observation of HDAC2-dependent sensitization of breast and pancreatic cancer cells towards topoisomerase II inhibitors [13,29], of breast cancer cells towards antihormonal therapy [30] and of colon cancer cells towards TNF $\alpha$ [31], characterizes HDAC2 as an important therapeutic target. Furthermore, HDAC2 contributes to EMT, one initial mechanism of metastasis, by the downregulation of Ecadherin in pancreatic cancer cells [32]. Altogether, these data may base the development of HDAC2 isoenzyme specific inhibitors with increased inhibitory capacity and lower toxicity compared to current HDACI [33].

Several TRAIL resistance mechanisms working at the level of the TRAIL receptors, the TRAIL DISC, the inhibitor of apoptosis proteins (IAPs) or the mitochondrium were described in PDAC cells $[18,19]$. However, consis- 


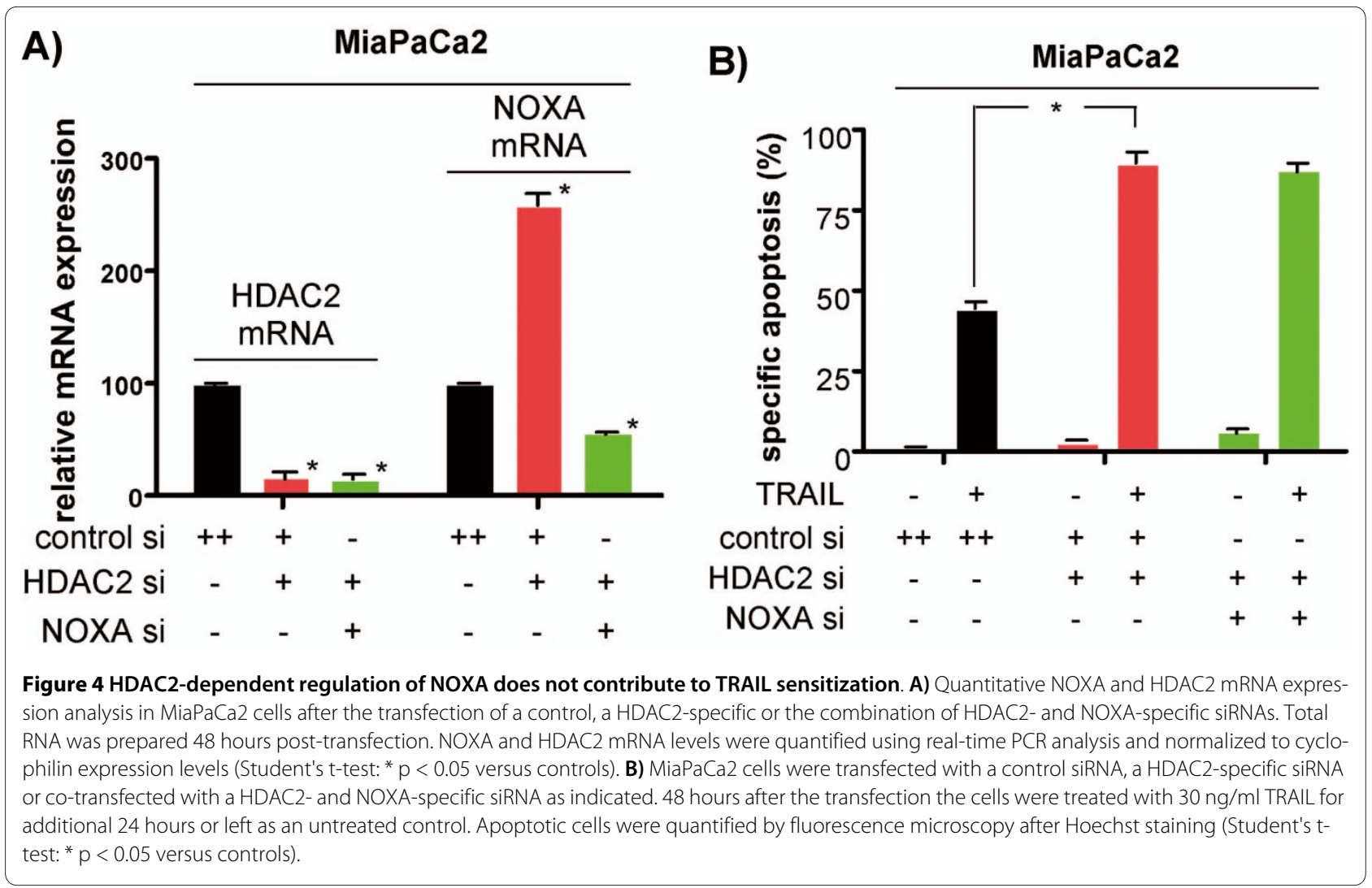

tent with our recent transcriptome profiling [13], we were not able to detect a clear downregulation of c-Flip, XIAP, cIAP1, cIAP2, $\mathrm{mcl}_{1}, \mathrm{bcl}_{\mathrm{XL}}$ or survivin the protein level after depletion of HDAC2, although an involvement of these proteins in the control of TRAIL resistance was described [26,34-43]. Using the HDACI butyrate, Natoni et al. described a HDAC-dependent control of sensitivity of PDAC cells towards Fas-induced apoptosis. At the molecular level the authors observed a reduced expression of $\mathrm{bcl}_{\mathrm{XL}}$ and c-Flip after the treatment with butyrate [44]. The failure to detect downregulation of $\mathrm{bcl}_{\mathrm{XL}}$ and cFlip after the sole HDAC2 depletion points to compensatory mechanisms working to maintain expression of these genes in PDAC cells. Nonetheless, our results are consistent with recent observations in HCT116 colon cancer cells, where HDACI treatment or depletion of HDAC2, but not HDAC1, sensitizes towards TNF $\alpha$-induced apoptosis [31]. Here, a contribution of NF- $\mathrm{kB}$ in the HDAC2dependent sensitization was proposed, since HDAC2 siRNAs reduce the TNF $\alpha$ mediated activation of a NF- $\mathrm{BB}$ luciferase reporter gene [31]. Consistently, a recent study reveals that treatment of the PDAC cell line Panc1 with the HDACI SAHA reduces basal binding of RelA to a consensus $\mathrm{kB}$ oligonucleotide [27]. Alike, we observed dependency of the basal NF- $\mathrm{KB}$ transcriptional activity on the presence of HDAC2 in PDAC cells (data not shown).
The observation that basal, but not induced, NF- $\mathrm{kB}$ activity contributes to TRAIL resistance of PDAC cells [45] and that inhibition of NF-kB by the super-inhibitor delta$\mathrm{N}-\mathrm{I}_{\kappa} \mathrm{B} \alpha$ [46] or a p65 siRNA [47] resulted in profound sensitization towards TRAIL-induced apoptosis in PDAC cells, might point to the contribution of NF- $\mathrm{KB}$ in our model system. However, we cannot rule out NF-kB-independent mechanisms at the moment and the ultimate clarification awaits further experiments beyond the scope of the article.

HDACI sensitize cancer cells towards TRAIL and several mechanisms, like upregulation of TRAIL and TRAIL receptors, downregulation of c-Flip, XIAP or members of the anti-apoptotic bcl2 family members as well as the modulation of NF-kB activity were shown to contribute $[48,49]$. As observed in many studies using HDACI to sensitize towards TRAIL, we observed a slight upregulation of DR5 protein abundance and cell surface expression in MiaPaCa2 cells after the depletion of HDAC2. The notice that HDACI-dependent TRAIL sensitization is independent of DR5 [50] and that DR5 regulation was not observed in Panc1 cells points to an alternative HDAC2 regulated mechanism contributing to the sensitization of PDAC cells. The demonstration of accelerated TRAIL-induced Bid cleavage after the depletion of HDAC2 argues that an event upstream of Bid is under 
A)

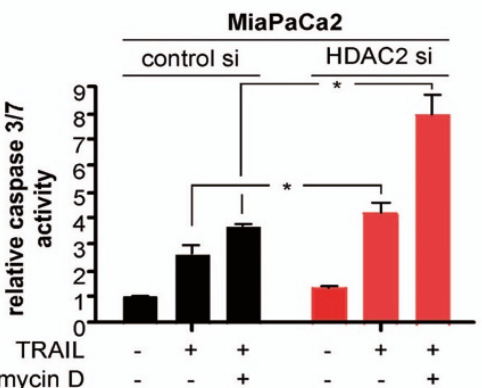

B) MiaPaCa2

TRAIL $(h)$

control si

HDAC2 si

$\begin{array}{llllll}0 & 1 & 3 & 0 & 1 & 3\end{array}$

$+++--$

$75 \mathrm{kD}-\ldots-\ldots-$ cleaved PARP

$50 \mathrm{kD}-\underset{-40 / 36 \mathrm{kD}}{-50 / 50 \mathrm{kD}}$

$25 \mathrm{kD}$

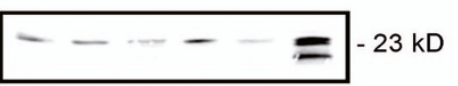

$50 \mathrm{kD}$
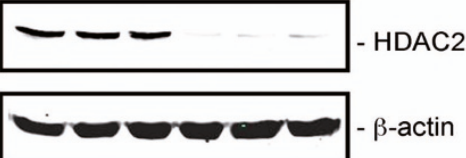

C)

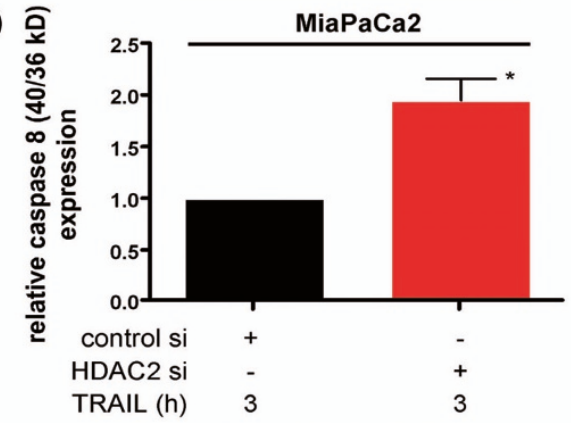

E) Panc1 MiaPaCa2

control si $+-\quad+\quad$

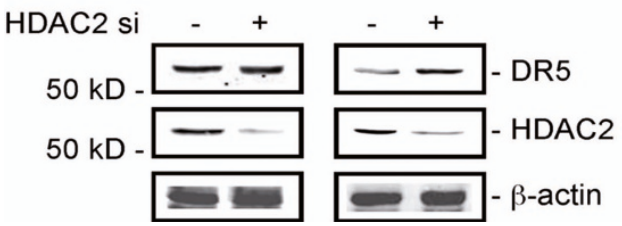

D)

MiaPaCa2

control si $+\quad+\quad-\quad-$

HDAC2 si - $\quad-\quad+\quad+$

TRAIL (h) $0 \quad 6 \quad 0 \quad 6$

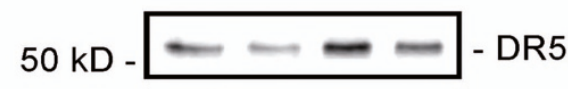

50 kD - - DR4

15 kD - $\longrightarrow-$ Bid

50 kD - - Flip $_{L}$

$50 \mathrm{kD}-\longrightarrow$ - XIAP

100 kD -

caspase 8

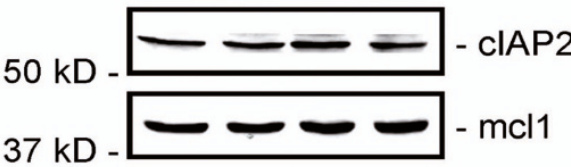

$25 \mathrm{kD}-\longleftarrow-$ bcl $_{\mathrm{XL}}$
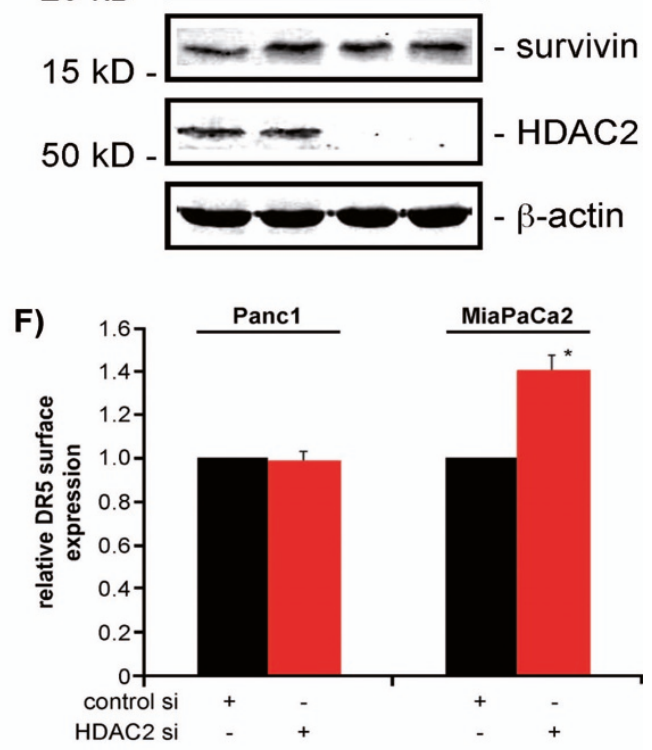

Figure 5 HDAC2-dependent regulation of DR5 in MiaPaCa2 cells. MiaPaCa2 cells were transfected with a control or a HDAC2-specific siRNA as indicated. A) 48 hours after the transfection the cells were treated with $15 \mathrm{ng} / \mathrm{ml}$ TRAlL or a combination of TRAlL and actinomycin D $(1 \mu \mathrm{g} / \mathrm{ml})$ for additional 2 hours. Afterwards caspase 3/7 activity was measured (Student's t-test: * $p<0.05$ versus controls). B) 48 hours after the transfection the cells were treated with $30 \mathrm{ng} / \mathrm{ml}$ TRAlL as indicated. Western blot determines caspase 8 (55/50 kD), caspase 8 cleavage products (migrating at 40/36 $\mathrm{kD}$ and $23 \mathrm{kD}$ ), cleaved PARP and HDAC2 expression. $\beta$-actin controls equal protein loading. C) MiaPaCa2 cells were treated as in B). The caspase 8 cleavage product migrating at 40/36 kD was quantified in control and HDAC2 siRNA transfected MiaPaCa2 cells 3 hours after the TRAIL treatment in four independent experiments (Student's t-test: ${ }^{*} p<0.05$ versus controls). D) 48 hours after the transfection the cells were treated with $30 \mathrm{ng} / \mathrm{ml}$ TRAIL for 6 hours. Western blot determines DR5, DR4, Bid, c-Flip, XIAP, CIAP1, cIAP2, mcl1, bcl ${ }_{\mathrm{XL}}$, survivin and HDAC2 expression. $\beta$-actin controls equal protein loading. E) Panc1 (left graph) and MiaPaCa2 cells (right graph) were transfected as indicated. Western blot determines DR5 and HDAC2 expression 48 hours after the transfection. $\beta$-actin controls equal protein loading. F) Panc1 (left graph) and MiaPaCa2 cells (right graph) were transfected as indicated. 48 hours after the transfection DR5 surface expression was determined by FACS analysis (Student's t-test: * $p<0.05$ versus controls). 
HDAC2 control in PDAC cells. Accordingly, HDACIdependent increased recruitment of the DISC component Fas-associated death domain protein (FADD) to the TRAIL-R1 was recently shown to play a role in the HDACI-mediated sensitization of CLL cells to TRAIL [51]. Although accelerated DISC formation and function after HDAC2 depletion is an attractive possibility, it is currently not known whether HDAC2 can control DISC formation and proof of this alternative explanation awaits additional experiments.

\section{Conclusions}

In summary, we have described a novel non-redundant HDAC2 function in PDAC cells that provides the rationale for further preclinical evaluation of a HDACI (HDAC2 isoenzyme-specific) and TRAIL combination therapy. Furthermore, our experiments point to a way to overcome TRAIL resistance of PDAC cells, needed to succeed with a TRAIL targeted therapy in clinical settings.

\section{Methods \\ Cell culture, siRNA transfection and reagents}

The pancreatic cancer cell lines MiaPaCa2 and Panc1 were cultivated as recently described [13]. Valproic acid (VPA) and TRAIL were purchased from EMD (EMD Biosciences, San Diego, CA, USA). Actinomycin D was from Sigma-Aldrich (Sigma-Aldrich, Munich, Germany). Untreated controls received vehicle alone. Doublestranded siRNAs were transfected at a final concentration of $50 \mathrm{nM}$ using oligofectamine (Invitrogen, Karlsruhe, Germany) according to the manufacturer's protocol. siRNAs were purchased from Eurofins, Ebersberg, Germany. Sequences of the used siRNAs were: control siRNA $5^{\prime} \mathrm{C}$ A G T C G C G T T T G C G A C T G G dtdt 3', HDAC2 siRNA 5' G C C T C A T A G A A T C C G C A T G dtdt 3 ', HDAC1 siRNA 5' G C A G A T G C A G A G A T T C A A C dtdt 3', NOXA siRNA 5' G G A A G T C G A G T G T G C T A C T dtdt 3'. For the simultaneous transfection of siRNAs directed against two different genes, the total amount of siRNA (100 nM) was kept constant using control siRNA.

\section{Statistical methods}

All data were obtained from at least three independent experiments performed in triplicate, and the results are presented as mean and standard error of the mean (S.E.M.). To demonstrate statistical significance a twotailed Student's t-test was used. p-values are indicated and * denotes a p-value of at least $<0.05$.

\section{Quantitative Reverse-Transcriptase PCR}

Total RNA was isolated from pancreatic carcinoma cell lines using the RNeasy kit (Qiagen, Hilden, Germany) fol- lowing the manufacturer's instructions. Quantitative mRNA analyses were performed as previously described using real-time PCR analysis (TaqMan, PE Applied Biosystems, Norwalk CT) [52,53]. Expression levels were normalized using cyclophilin. Primer sequences were as follows: cyclophilin-fw 5 A T G G T C A A C C C C A C C G T G T 3'; cyclophilin-rev 5' T C T G C T G T C T T T G G G A C C T T G T C 3'; HDAC2-fw 5' A G C A T C A G G A T T C T G T T A C G T T A A T G A 3'; HDAC2rv5' C A A C A C C A T C A C C A T G A T G A A T A T C T 3'; NOXA-fw 5' C G G A G A T G C C T G G G A A G A A 3'; NOXA-rev 5' C C A A A T C T C C T G A G T T G A G T A G C A 3'.

\section{Total cell lysates and Western blot}

Whole cell lysates were prepared and western blots were done as recently described [52,53]. The following antibodies were used: DR4, DR5 (ProScience Inc., Poway, CA, USA); HDAC2 (H-54, sc-7899) (Santa Cruz Biotechnology, Santa Cruz, CA, USA); XIAP, cIAP1, cIAP2, survivin (R\&D Systems, Minneapolis, MN, USA); Bid, bcl $\mathrm{XL}_{\mathrm{LL}}$ (Cell Signaling Technology Inc., Danvers, MA, USA); NOXA, mcl1 (Alexis Biochemicals, San Diego, CA, USA); c-Flip, cleaved PARP, caspase 8 (BD Biosciences, Heidelberg, Germany); HDAC1 (Upstate/Millipore, Billerica, MA, USA); $\beta$-actin (Sigma-Aldrich, Munich, Germany). One representative western blot out of at least three independent experiments is shown. Western blots were quantified using Odyssey Infrared Imaging System (LI-COR Biosciences, Bad Homburg, Germany), assuring measurements in the linear range.

\section{MTT- and Caspase 3/7-assay}

Viability of the cells was measured using MTT-assays performed according to the manufacturer's protocol (Roche Applied Science, Mannheim, Germany). Caspase 3/7 activity was determined using Promega's CaspaseGlo 3/7 assay according to the manufacturer's instructions (Promega, Madison, WI, USA).

\section{Analysis of cell surface expression of DR5}

Cells were transfected as indicated. After $48 \mathrm{~h}$ cells were trypsinized, washed once with PBS and suspended in PBS containing $2 \mu \mathrm{g} / \mathrm{ml}$ primary antibody (anti-DR5; R\&D Systems, Minneapolis, USA) or control IgG. Cells were stained on ice for $60 \mathrm{~min}$, then washed with $3 \mathrm{ml}$ cold PBS and incubated with the secondary antibody (1:100 dilution PE-conjugated goat anti-mouse $\mathrm{F}\left(\mathrm{ab}^{\prime}\right) 2$ antiIgG+IgM (Jackson ImmunoResearch, Suffolk, England)) for $60 \mathrm{~min}$, on ice, in the dark. After an additional wash with $3 \mathrm{ml}$ cold PBS, cells were suspended in $1 \mathrm{ml}$ cold FACS buffer and 10.000 cells per sample were analyzed using fluorescence flow cytometry (Galaxy Argon Plus, 
Dako, Glostrup, Denmark) and results were analysed with the FLOMAX software (Dako).

\section{Competing interests}

The authors declare that they have no competing interests.

\section{Authors' contributions}

$S S, P F, S D, A A, D S$, and GS designed and conducted the experiments. SS, PF, SD, $\mathrm{AA}, \mathrm{DS}$, and GS analyzed and interpreted data. SS, PF, SD, AA, DS, RMS and GS drafted the manuscript and revised it critically for important intellectual content. All authors read and approved the final manuscript.

\section{Acknowledgements}

This work was supported by Deutsche Forschungsgemeinschaft (Grant SCHN 959/1-2; SFB456), Else Kröner-Fresenius-Stiftung, Fritz-Thyssen Stiftung, Bayerische Forschungsstiftung. We would like to thank Mses. Hoffmann and Kohnke-Ertel for excellent technical support.

\section{Author Details}

'Technische Universität München, Klinikum rechts der Isar, II. Medizinische Klinik, Ismaninger Str. 22, 81675 Munich, Germany and 2Christian-AlbrechtsUniversität Kiel, Department of Internal Medicine, University-Hospital Schleswig-Holstein (UKSH)-Campus Kiel, Schittenhelmstr. 12, 24105 Kiel, Germany

Received: 20 November 2009 Accepted: 16 April 2010 Published: 16 April 2010

\section{References}

1. Schneider G, Siveke JT, Eckel F, Schmid RM: Pancreatic cancer: basic and clinical aspects. Gastroenterology 2005, 128:1606-1625.

2. Mitry E, Rachet B, Quinn MJ, Cooper N, Coleman MP: Survival from cancer of the pancreas in England and Wales up to 2001. Br J Cancer 2008, 99(Suppl 1):S21-23.

3. Moore MJ, Goldstein D, Hamm J, Figer A, Hecht JR, Gallinger S, Au HJ, Murawa $P$, Walde D, Wolff RA, et al:: Erlotinib plus gemcitabine compared with gemcitabine alone in patients with advanced pancreatic cancer: a phase III trial of the National Cancer Institute of Canada Clinical Trials Group. J Clin Oncol 2007, 25:1960-1966.

4. Yang XJ, Seto E: The Rpd3/Hda1 family of lysine deacetylases: from bacteria and yeast to mice and men. Nat Rev Mol Cell Biol 2008, 9:206-218.

5. Buchwald $\mathrm{M}$, Krämer $\mathrm{OH}$, Heinzel T: HDACi--targets beyond chromatin. Cancer Lett 2009, 280:160-167.

6. Spange $\mathrm{S}$, Wagner T, Heinzel T, Krämer OH: Acetylation of non-histone proteins modulates cellular signalling at multiple levels. Int J Biochem Cell Biol 2009, 41:185-198.

7. Witt O, Deubzer HE, Milde T, Oehme I: HDAC family: What are the cancer relevant targets? Cancer Lett 2009, 277:8-21.

8. Glozak MA, Seto E: Histone deacetylases and cancer. Oncogene 2007, 26:5420-5432.

9. Schneider G, Krämer OH, Fritsche P, Schüler S, Schmid RM, Saur D: Targeting histone deacetylases in pancreatic ductal adenocarcinoma. $J$ Cell Mol Med 2009 in press.

10. Marks PA, Xu WS: Histone deacetylase inhibitors: Potential in cancer therapy. J Cell Biochem 2009, 107:600-8.

11. Bots M, Johnstone RW: Rational combinations using HDAC inhibitors. Clin Cancer Res 2009, 15:3970-3977.

12. Richards DA, Boehm KA, Waterhouse DM, Wagener DJ, Krishnamurthi SS, Rosemurgy A, Grove W, Macdonald K, Gulyas S, Clark M, Dasse KD: Gemcitabine plus $\mathrm{Cl}-994$ offers no advantage over gemcitabine alone in the treatment of patients with advanced pancreatic cancer: results of a phase II randomized, double-blind, placebo-controlled, multicenter study. Ann Oncol 2006, 17:1096-1102.

13. Fritsche P, Seidler B, Schüler S, Schnieke A, Göttlicher M, Schmid RM, Saur D, Schneider G: HDAC2 mediates therapeutic resistance of pancreatic cancer cells via the BH3-only protein NOXA. Gut 2009, 58:1399-1409.

14. Göttlicher M, Minucci S, Zhu P, Kramer OH, Schimpf A, Giavara S, Sleeman JP, Lo Coco F, Nervi C, Pelicci PG, Heinzel T: Valproic acid defines a novel class of HDAC inhibitors inducing differentiation of transformed cells. Embo J 2001, 20:6969-6978.
15. Krämer OH, Zhu P, Ostendorff HP, Golebiewski M, Tiefenbach J, Peters MA, Brill B, Groner B, Bach I, Heinzel T, Göttlicher M: The histone deacetylase inhibitor valproic acid selectively induces proteasomal degradation of HDAC2. Embo J 2003, 22:3411-3420.

16. Igney $\mathrm{FH}, \mathrm{Krammer} \mathrm{PH}$ : Death and anti-death: tumour resistance to apoptosis. Nat Rev Cancer 2002, 2:277-288.

17. Fulda S, Debatin KM: Extrinsic versus intrinsic apoptosis pathways in anticancer chemotherapy. Oncogene 2006, 25:4798-4811.

18. Hamacher R, Schmid RM, Saur D, Schneider G: Apoptotic pathways in pancreatic ductal adenocarcinoma. Mol Cancer 2008, 7:64.

19. Fulda S: Apoptosis pathways and their therapeutic exploitation in pancreatic cancer. J Cell Mol Med 2009, 13:1221-1227.

20. Ashkenazi A: Directing cancer cells to self-destruct with pro-apoptotic receptor agonists. Nat Rev Drug Discov 2008, 7:1001-1012.

21. Newsom-Davis T, Prieske S, Walczak H: Is TRAIL the holy grail of cancer therapy? Apoptosis 2009, 14:607-623.

22. Sanlioglu AD, Dirice E, Elpek O, Korcum AF, Balci MK, Omer A, Griffith TS, Sanlioglu S: High levels of endogenous tumor necrosis factor-related apoptosis-inducing ligand expression correlate with increased cell death in human pancreas. Pancreas 2008, 36:385-393.

23. Sanlioglu AD, Dirice E, Elpek O, Korcum AF, Ozdogan M, Suleymanlar I, Balci MK, Griffith TS, Sanlioglu S: High TRAIL death receptor 4 and decoy receptor 2 expression correlates with significant cell death in pancreatic ductal adenocarcinoma patients. Pancreas 2009, 38:154-160.

24. Ozawa F, Friess H, Kleeff J, Xu ZW, Zimmermann A, Sheikh MS, Büchler MW: Effects and expression of TRAIL and its apoptosis-promoting receptors in human pancreatic cancer. Cancer Lett 2001, 163:71-81.

25. Koschny R, Walczak H, Ganten TM: The promise of TRAIL--potential and risks of a novel anticancer therapy. J Mol Med 2007, 85:923-935.

26. Vogler M, Durr K, Jovanovic M, Debatin KM, Fulda S: Regulation of TRAILinduced apoptosis by XIAP in pancreatic carcinoma cells. Oncogene 2007, 26:248-257.

27. Lehmann A, Denkert C, Budczies J, Buckendahl AC, Darb-Esfahani S, Noske A, Muller BM, Bahra M, Neuhaus P, Dietel M, et al:: High class I HDAC activity and expression are associated with RelA/p65 activation in pancreatic cancer in vitro and in vivo. BMC Cancer 2009, 9:395.

28. Weichert W: HDAC expression and clinical prognosis in human malignancies. Cancer Lett 2009, 280:168-176.

29. Marchion DC, Bicaku E, Turner JG, Schmitt ML, Morelli DR, Munster PN: HDAC2 regulates chromatin plasticity and enhances DNA vulnerability. Mol Cancer Ther 2009, 8:794-801.

30. Bicaku E, Marchion DC, Schmitt ML, Munster PN: Selective inhibition of histone deacetylase 2 silences progesterone receptor-mediated signaling. Cancer Res 2008, 68:1513-1519.

31. Kaler P, Sasazuki T, Shirasawa S, Augenlicht L, Klampfer L: HDAC2 deficiency sensitizes colon cancer cells to TNFalpha-induced apoptosis through inhibition of NF-kappaB activity. Exp Cell Res 2008, 314:1507-1518.

32. von Burstin J, Eser S, Paul MC, Seidler B, Brandl M, Messer M, von Werder A, Schmidt A, Mages J, Pagel P, et al.: E-cadherin regulates metastasis of pancreatic cancer in vivo and is suppressed by a SNAIL/HDAC1/HDAC2 repressor complex. Gastroenterology 2009, 137:361-371. 371 e361-365

33. Krämer OH: $\mathrm{HDAC2}$ : a critical factor in health and disease. Trends Pharmacol Sci 2009, 30:647-55.

34. Bai J, Sui J, Demirjian A, Vollmer CM Jr, Marasco W, Callery MP: Predominant Bcl-XL knockdown disables antiapoptotic mechanisms: tumor necrosis factor-related apoptosis-inducing ligand-based triple chemotherapy overcomes chemoresistance in pancreatic cancer cells in vitro. Cancer Res 2005, 65:2344-2352.

35. Retzer-Lidl M, Schmid RM, Schneider G: Inhibition of CDK4 impairs proliferation of pancreatic cancer cells and sensitizes towards TRAILinduced apoptosis via downregulation of survivin. Int J Cancer 2007, 121:66-75.

36. Song JJ, An JY, Kwon YT, Lee YJ: Evidence for two modes of development of acquired tumor necrosis factor-related apoptosisinducing ligand resistance. Involvement of Bcl-xL. J Biol Chem 2007, 282:319-328.

37. Wang P, Zhang J, Bellail A, Jiang W, Hugh J, Kneteman NM, Hao C: Inhibition of RIP and c-FLIP enhances TRAIL-induced apoptosis in pancreatic cancer cells. Cell Signal 2007, 19:2237-2246.

38. Vogler M, Walczak H, Stadel D, Haas TL, Genze F, Jovanovic M, Gschwend JE, Simmet T, Debatin KM, Fulda S: Targeting XIAP bypasses BCl-2- 
mediated resistance to TRAIL and cooperates with TRAIL to suppress pancreatic cancer growth in vitro and in vivo. Cancer Res 2008, 68:7956-7965.

39. Vogler M, Walczak H, Stadel D, Haas TL, Genze F, Jovanovic M, Bhanot U, Hasel C, Moller P, Gschwend JE, et al:: Small molecule XIAP inhibitors enhance TRAIL-induced apoptosis and antitumor activity in preclinical models of pancreatic carcinoma. Cancer Res 2009, 69:2425-2434.

40. Mori T, Doi R, Kida A, Nagai K, Kami K, Ito D, Toyoda E, Kawaguchi Y, Uemoto S: Effect of the XIAP inhibitor Embelin on TRAIL-induced apoptosis of pancreatic cancer cells. J Surg Res 2007, 142:281-286.

41. Huang S, Sinicrope FA: BH3 mimetic ABT-737 potentiates TRAILmediated apoptotic signaling by unsequestering Bim and Bak in human pancreatic cancer cells. Cancer Res 2008, 68:2944-2951.

42. Huang S, Okumura K, Sinicrope FA: BH3 mimetic obatoclax enhances TRAIL-mediated apoptosis in human pancreatic cancer cells. Clin Cancer Res 2009, 15:150-159.

43. Murtaza I, Saleem M, Adhami VM, Hafeez BB, Mukhtar H: Suppression of cFLIP by lupeol, a dietary triterpene, is sufficient to overcome resistance to TRAIL-mediated apoptosis in chemoresistant human pancreatic cancer cells. Cancer Res 2009, 69:1156-1165.

44. Natoni F, Diolordi L, Santoni C, Gilardini Montani MS: Sodium butyrate sensitises human pancreatic cancer cells to both the intrinsic and the extrinsic apoptotic pathways. Biochim Biophys Acta 2005, 1745:318-329.

45. Braeuer SJ, Buneker C, Mohr A, Zwacka RM: Constitutively activated nuclear factor-kappaB, but not induced NF-kappaB, leads to TRAIL resistance by up-regulation of $X$-linked inhibitor of apoptosis protein in human cancer cells. Mol Cancer Res 2006, 4:715-728.

46. Trauzold A, Wermann H, Arlt A, Schutze S, Schafer H, Oestern S, Roder C, Ungefroren $\mathrm{H}$, Lampe $\mathrm{E}$, Heinrich M, et al: CD95 and TRAIL receptormediated activation of protein kinase $\mathrm{C}$ and $\mathrm{NF}$-kappaB contributes to apoptosis resistance in ductal pancreatic adenocarcinoma cells. Oncogene 2001, 20:4258-4269.

47. Khanbolooki S, Nawrocki ST, Arumugam T, Andtbacka R, Pino MS, Kurzrock R, Logsdon CD, Abbruzzese JL, McConkey DJ: Nuclear factorkappaB maintains TRAIL resistance in human pancreatic cancer cells. Mol Cancer Ther 2006, 5:2251-2260.

48. Fulda S: Modulation of TRAIL-induced apoptosis by HDAC inhibitors. Curr Cancer Drug Targets 2008, 8:132-140.

49. Carew JS, Giles FJ, Nawrocki ST: Histone deacetylase inhibitors: mechanisms of cell death and promise in combination cancer therapy. Cancer Lett 2008, 269:7-17.

50. Inoue S, Twiddy D, Dyer MJ, Cohen GM: Upregulation of TRAIL-R2 is not involved in HDACi mediated sensitization to TRAIL-induced apoptosis. Cell Death Differ 2006, 13:2160-2162.

51. Inoue S, Harper N, Walewska R, Dyer MJ, Cohen GM: Enhanced Fasassociated death domain recruitment by histone deacetylase inhibitors is critical for the sensitization of chronic lymphocytic leukemia cells to TRAIL-induced apoptosis. Mol Cancer Ther 2009, 8:3088-3097.

52. Reichert M, Saur D, Hamacher R, Schmid RM, Schneider G: Phosphoinositide-3-kinase signaling controls S-phase kinaseassociated protein 2 transcription via E2F1 in pancreatic ductal adenocarcinoma cells. Cancer Res 2007, 67:4149-4156.

53. Schneider G, Henrich A, Greiner G, Wolf V, Lovas A, Wieczorek M, Wagner T, Reichardt S, von Werder A, Schmid RM, et al: Cross talk between stimulated NF-kappaB and the tumor suppressor p53. Oncogene 2010 in press.

doi: $10.1186 / 1476-4598-9-80$

Cite this article as: Schüler et al., HDAC2 attenuates TRAIL-induced apoptosis of pancreatic cancer cells Molecular Cancer 2010, 9:80

\section{Submit your next manuscript to BioMed Central} and take full advantage of:

- Convenient online submission

- Thorough peer review

- No space constraints or color figure charges

- Immediate publication on acceptance

- Inclusion in PubMed, CAS, Scopus and Google Scholar

- Research which is freely available for redistribution

Submit your manuscript at www.biomedcentral.com/submit
C Biomed Central 